\title{
Warburg effect, hexokinase-II, and radioresistance of laryngeal carcinoma
}

\author{
Jiang-Tao Zhong ${ }^{1}$ and Shui-Hong Zhou ${ }^{1}$ \\ ${ }^{1}$ Department of Otolaryngology, The First Affiliated Hospital, College of Medicine, Zhejiang University, Hangzhou, Zhejiang, \\ China \\ Correspondence to: Shui-Hong Zhou, email: zhoushuihong2016@sina.com
}

Keywords: hexokinase-ll, Warburg effect, laryngeal carcinoma, radioresistance

Received: May 20, 2016

Accepted: October 28, 2016

Published: November 03, 2016

\section{ABSTRACT}

Radiotherapy is now widely used as a part of multidisciplinary treatment approaches for advanced laryngeal carcinoma and preservation of laryngeal function. However, the mechanism of the radioresistance is still unclear. Some studies have revealed that the Warburg effect promotes the radioresistance of various malignant tumors, including laryngeal carcinoma. Among the regulators involved in the Warburg effect, hexokinase-II (HK-II) is a crucial glycolytic enzyme that catalyzes the first essential step of glucose metabolism. HK-II is reportedly highly expressed in some human solid carcinomas by many studies. But for laryngeal carcinoma, there is only one. Till now, no studies have directly targeted inhibited HK-II and enhanced the radiosensitivity of laryngeal carcinoma. Accumulating evidence has shown that dysregulated signaling pathways often result in HK-II overexpression. Here, we summarize recent advances in understanding the association among the Warburg effect, HK-II, and the radioresistance of laryngeal carcinoma. We speculate on the feasibility of enhancing radiosensitivity by targeted inhibiting HK-II signaling pathways in laryngeal carcinoma, which may provide a novel anticancer therapy.

\section{INTRODUCTION}

Approximately 157,000 new cases of laryngeal carcinoma were diagnosed worldwide in 2012 (1\% of all cancers), and approximately 83,400 people were estimated to have died from laryngeal carcinoma in that year $(1 \%$ of all cancer deaths) [1]. Previously, total laryngectomy was the mainstay of treatment for laryngeal carcinoma, which was successfully performed for the first time in 1873 by Theodor Billroth [2]. Although this procedure decreases the recurrence rate of laryngeal carcinoma, it leaves patients with postoperative aphonia and reliance on a permanent tracheostoma. Nowadays, with the evolution of treatment for advanced laryngeal carcinoma, the focus of treatment has shifted to maintaining locoregional control while also maintaining a functional larynx [3]. Therefore, multidisciplinary treatment approaches are recommended. As radiotherapy (RT) techniques have markedly improved recently, the incidence of laryngeal function preservation in laryngeal carcinoma has increased [4-7]. However, RT results in acute toxicities including mucositis, dysphagia, xerostomia, dermatitis, and pain.
In addition, radioresistance has been found in laryngeal carcinoma. However, the exact mechanisms behind this radioresistance have not been clarified. The mechanisms may include cancer stem cells [8-11], hypoxia [e.g., Hypoxia-inducible factor $1 \alpha(\mathrm{HIF}-1 \alpha)$ ] [12], human papillomavirus (HPV) [13], and signal transduction pathways [e.g., the signal transducer and activator of transcription 3 (STAT3), phosphoinositide-3 kinase (PI3K)/Akt].

In recent years, the Warburg effect (aerobic glycolysis) has been widely investigated. It is an anomalous characteristic of cancer cells, in which they consume a large amount of glucose and favor aerobic glycolysis over oxidative phosphorylation even in the presence of oxygen. A few studies have demonstrated that the Warburg effect contributes to the radioresistance of various malignant tumors [12, 14-20]. Inhibition of the Warburg effect has been shown to enhance the radiosensitivity of cancers [14, 21-23]. Similar results have also been found in laryngeal carcinoma [24-29].

Hexokinase-II (HK-II) is the enzyme that catalyzes the first essential step of glucose metabolism, 
the conversion of glucose into glucose-6-phosphate, in the Warburg effect [30]. HK-II has been found highly expressed in many different cancers \{e.g., laryngeal carcinoma [31], oral squamous cell carcinoma [32], hepatocellular carcinoma (HCC) $[33,34]$, breast cancer [35], colorectal tumors [36], etc. . Several studies have demonstrated that HK-II upregulation not only increases the glycolysis rate, but is also required for tumor initiation and maintenance [37]. For example, voltage-dependent anion channel (VDAC)-bound HK-II contributes to the inhibition of apoptosis by suppressing the formation of mitochondrial permeability transition pores (mPTPs) [38]. On the other hand, numerous studies have indicated that inhibitors [e.g., 2-deoxyglucose (2-DG), lonidamine, 3-bromopyruvate (3-BP)] targeted inhibiting HK-II can induce cancer cells to undergo apoptosis [39]. To date, only one study has reported HK-II overexpression in laryngeal carcinoma [31], while another revealed that HK-II depletion sensitizes radioresistant Hep-2 cells to radiation, although the result did not reach significance [40]. As such, the association between HK-II and radioresistance in laryngeal carcinoma requires further investigation.

In this review, we discuss the role of the Warburg effect and HK-II in the radioresistance of laryngeal carcinoma, and the feasibility of enhancing radiosensitivity by targeted inhibiting the signaling pathways of HK-II in laryngeal carcinoma, which may be a novel anticancer therapy.

\section{RADIORESISTANCE OF LARYNGEAL CARCINOMA}

Many studies have demonstrated that cancer stem cells, signal transduction pathways, and hypoxia are vital for the radioresistance of laryngeal carcinoma $[26,41-50]$. These roles have also been reported in our previous reviews [25, 51]. Additional factors involved in radioresistance have also been identified, including changes in DNA levels and ultrastructure.

With regard to changes in DNA levels potentially mediating the radioresistance of cancer cells, Kim et al. found that the promoter-CpG islands of five previously identified radioresistance-related genes (TOPO2A, PLXDC2, ETNK2, GFI1, and IL12B) were significantly altered in radioresistant laryngeal carcinoma. This was determined by examining the differences in DNA methylation between control and radioresistant laryngeal carcinoma, the latter of which had been established by long-term fractionated irradiation. In addition, the demethylation of these gene promoters via a DNA methyltransferase inhibitor (5-aza-2'-deoxycytidine) was shown to increase their transcription levels. This study suggests that radiation-induced epigenetic changes can stimulate the radioresistance of laryngeal carcinoma [52]. Furthermore, the PAG1 gene has also been identified as a promising novel radiosensitization target for laryngeal carcinoma. Ke et al. used small interfering RNA (siRNA) to targeted suppress the PAG1 gene in a radioresistant cell line and dramatically enhanced its radiosensitivity and irradiation-induced cell death, while the ectopic expression of PAG1 in radiosensitive cell lines led to radioresistance and suppressed irradiation-induced cell death. These results demonstrate that PAG1 acts as a radioresistance factor in laryngeal carcinoma cells [53].

In a recent study, Yang et al. identified ultrastructural changes in the radiation-induced radioresistant laryngeal carcinoma Hep-2 (Hep-2R) cell line. Specifically, they observed increased nuclear atypia, more rough endoplasmic reticulum (ER), and fewer mitochondria in Hep-2R cells, which showed significant resistance to radiation compared with parental Hep-2 cells. This study indicated that ultrastructural changes are the morphological mechanism by which the radioresistance of Hep-2R cells is enhanced [54].

\section{AGENTS TARGETED INHIBITING THE HK-II-MEDIATED WARBURG EFFECT}

Some studies have demonstrated that agents targeted inhibiting HK-II may have an anticancer effect.

\section{2-DG}

2-DG is a glucose analogue and a competitive inhibitor of HK-II. It is phosphorylated by HK-II but not further processed by glucose 6-phosphate isomerase, resulting in the accumulation of phosphorylated 2-deoxyglucose (P-2-DG) in the cell and its competitive inhibition of HK-II by negative feedback. 2-DG has reached the phase I/II clinical trial stage for the treatment of human cerebral gliomas [55] and phase I clinical trials for prostate cancer patients [56]. Previously, Wang et al. found that the combination of 2-DG with molecule US597 (UA-4, a structurally modified version of ursolic acid) synergistically inhibited hepatoma cell proliferation by dual targeting of apoptosis and glycolysis [57]. Subsequently, they found that 2-DG may inhibit hepatocarcinogenesis in DEN-treated rats by restricting cancer cell metabolism [58].

\section{3-BP}

In addition to 2-DG, 3-BP, a halogenated analog of pyruvic acid, can also inhibit HK-II function in aerobic glycolysis, which promotes tumor cell death by inducing ER stress in human HCC cell lines. An early study showed that 3-BP exhibits a strong anti-glycolytic effect on rat mammary tumor cells implanted in rats [59].

For HCC, the inhibition of carbonic anhydraseIX (CA-IX) enhances the effect of 3-BP by aggravating 
ER stress and activating Jun NH2-terminal kinase (JNK) [60]. Another study revealed that combination therapy with 3-BP and a protein disulfide isomerase inhibitor (bacitracin) may be therapeutically useful in HCC [61]. A more recent study demonstrated that 3-BP exerts anti-hepatoma effects, as shown by in vitro and in vivo analyses [62]. In addition, in multiple myeloma (MM) cells, in which HK-II was over-expressed, 3-BP promptly and substantially suppressed adenosine triphosphate production and induced cell death [63]. For endometrial cancer, 3-BP induced tumor necrosis in vitro and inhibited tumor growth in vivo [64]. It has also been reported that 3-BP induced apoptosis in MDA-MB-231 breast cancer cells by downregulating Mcl-1 through the PI3K/Akt signaling pathway, which is upstream of HK-II [65]. Recently, Gandham et al. also showed that liposomal 3-BP improved permeability, HK-II inhibition, and cytotoxicity in a multicellular spheroid model of human ovarian adenocarcinoma (SKOV-3) cells $[66,67]$. However, because of its off-target toxicity, 3-BP has not yet been widely used for anticancer therapy.

\section{Lonidamine}

Lonidamine is a new drug that interferes with mitochondrial functions, which can inhibit HK-II function. It may be a useful tool for adversely affecting tumors via aerobic glycolysis [68]. Lonidamine has been evaluated in a phase II study, in combination with diazepam, for recurrent glioblastoma multiforme (GBM) [69]. It has also been used in other clinical trials for breast cancer, ovarian cancer, lung cancer, and prostate adenoma.

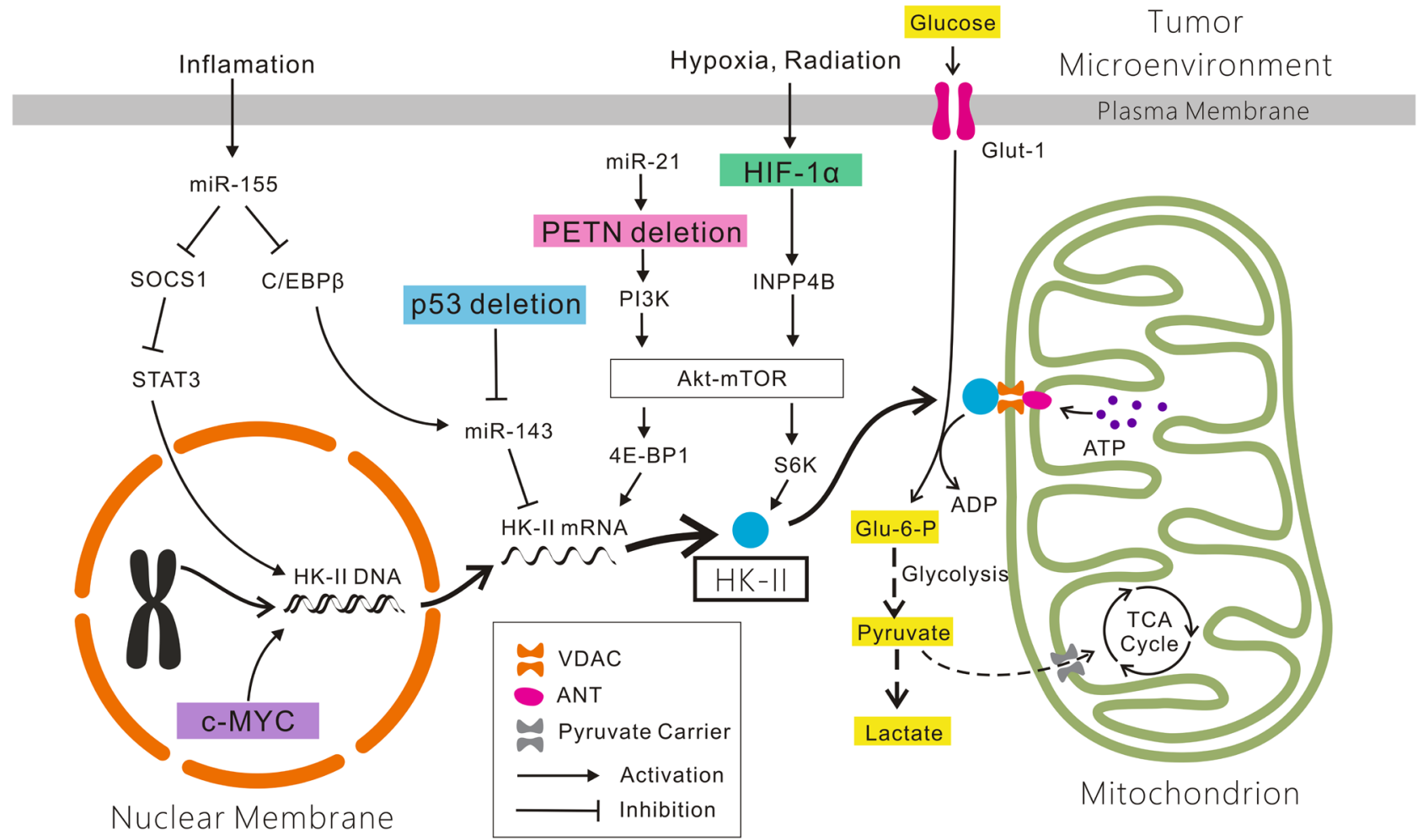

Figure 1: Mechanisms account for the altered expression of HK-II in cancer cells. Glucose which across the plasma membrane via Glut-1 is phosphorylated by HK-II bound to VDAC located on the outer mitochondrial membrane. The ATP involved in the phosphorylation of glucose is transfered by ANT through the HK-II/VDAC complex. The product Glu-6-P enters into the pentose phosphate shunt for biosynthesis of nucleic acid precursors (not shown), and the glycolytic pathway. Most pyruvate is reduced to lactate and some is directed to mitochondria via the pyruvate carrier for the TCA cycle for energy generation and biosynthesis. The HIF-1 $\alpha / \mathrm{INPP} 4 \mathrm{~B} /$

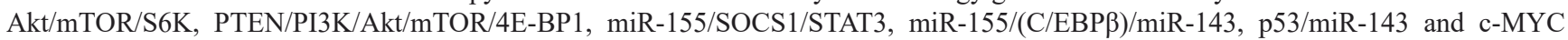
signaling pathways are showed here. See details in the text. Abbreviations: HK-II: Hexokinase-II; Glut-1: glucose transporter-1; VDAC: voltage-dependent anion channel; ANT: adenine nucleotide translocator; Glu-6-P: Glucose-6-P; TCA cycle: tri-carboxylic acid cycle. 


\section{Targeted inhibiting the HK-II-VDAC complex}

Mitochondrion-associated HK-II correlates strongly with the Warburg effect. The association between HKII and the mitochondrial membrane is mediated by VDAC. The HK-II-VDAC complex functions not only in glycolysis and biosynthesis, but also in the inhibition of mitochondrion-induced apoptosis and the suppression of cell death. Conversely, inhibition of the HK-II-VDAC complex significantly enhances the induction of apoptosis in tumors [70]. The binding of HK to VDAC prevents the localization of other activated pro-apoptotic molecules (e.g., Bad, Bax) to the outer mitochondrial membrane and also prevents formation of the mPTPs complex, thereby avoiding mitochondrial cytochrome c-mediated apoptosis [71].

Recent studies have identified several agents that targeted inhibit the HK-II-VDAC complex, which is regarded as a promising mitochondrial target as it is linked to glycolysis, oxidative phosphorylation, and mitochondrion-mediated apoptosis in tumor cells [72]. 3-BP, which has been discussed above, dissociates HKII from the HK-II-VDAC complex, causing tumor cell death. Some natural compounds have been shown to promote the detachment of HK-II from mitochondria, such as the natural plant flavonoid (oroxylin A), the plant hormones [methyl jasmonate (MJ), neoalbaconol (NA), and prosapogenin A] [73], and other synthetic compounds (casiopeina II-gly, clotrimazole, and bifonazole). Clotrimazole and bifonazole, which are azole derivatives, have been reported to sensitize glioblastoma cells to radiation by displacing HK-II from the mitochondrial membrane [74]. HK-II-targeted small hairpin RNA (shRNA) has also been shown to produce pronounced anti-tumor effects. The evidence above indicates that abrogation of the HK-II-VDAC complex may result in tumor cell apoptosis, thus increasing the sensitization of tumor cells to RT [38].

\section{Metformin}

Metformin, an anti-hyperglycemic drug, has also been revealed to exhibit important anticancer properties. One study showed that it impairs the enzymatic function of HK-II in Calu-1 cells, thus abolishing cell glucose uptake and phosphorylation, which results in mitochondrial depolarization and subsequent cell death [75]. A similar phenomenon was also found in breast cancer [35].

\section{HK-II siRNA}

siRNA have been used conventionally in basic research to silence target genes and observe the associated functional changes. Knockdown of HK-II with siRNA reduces its mRNA and protein levels, as well as its activity, resulting in cell cycle arrest at the G1 phase, attenuated glycolysis, and apoptosis in cancer cells [31, 76-78]. Silencing of the HK-II gene sensitizes human colon cancer cells to 5-fluorouracil [79] and sensitizes parental lung cancer cells to cisplatin [80]. In addition, the combination of HK-II interference and ${ }^{131} \mathrm{I}$ therapy demonstrated a stronger anticancer effect in anaplastic thyroid carcinoma cells [81]. However, further investigations of treatments with HK-II siRNA for laryngeal carcinoma are required.

\section{THE WARBURG EFFECT AND RADIORESISTANCE}

\section{The Warburg effect and radioresistance of cancer}

The Warburg effect is significant for cancer progression. It provides a survival advantage and facilitates the synthesis of biosynthetic precursors required for continued cellular proliferation.

Bhatt et al. found that mitochondrial respiratory modifiers [e.g., dinitrophenol (DNP)] induced the transient stimulation of glycolysis in the malignant cell lines BMG-1 and OCT-1, conferring radioresistance to the cells. They also demonstrated that the radioresistance of cells induced by DNP was obtained by reducing residual DNA damage and cytogenetic damage linked to mitotic death via the non-homologous end joining and homologous recombination pathways [16]. Tumor hypoxia is a common phenomenon revealed to be an important effector promoting tumor cell survival. A clinical study demonstrated that hypoxia is associated with the radioresistance of prostate cancer [12]. A similar study showed that hypoxia and anaerobic metabolism may be important components of prostate cancer radioresistance [18]. Hypoxia-inducible factor 1 (HIF-1) is important in the Warburg effect; it becomes active not only under hypoxic, but also normoxic, conditions. Accumulating evidence shows that HIF-1 functions in the induction of radioresistant characteristics in cancer cells and tumor recurrence after radiation therapy [15]. In addition, glucose transporter-1 (Glut-1), which also correlates strongly with the Warburg effect, was found to be associated with the radioresistance of breast cancer cells [14]. Akt regulates multiple steps in the Warburg effect, including inducing Glut-1 gene expression and enhancing HK activity. Shimura et al. showed that an Akt-mediated process enhanced aerobic glycolysis in the acquisition of radioresistance by tumor cells [17]. Signal transducer and activator of transcription 1 (STAT1), which is usually regarded as a transmitter of interferon signaling and a pro-apoptotic tumor suppressor, has also been found to be associated with the Warburg effect. Pitroda et al. found that activation of STAT1 enhances the radioresistance of 
tumors [20]. Furthermore, radioresistance was found to be positively correlated with the lactate concentration [19]. It has been reported that rapamycin can decrease production of lactate, which reverses pseudo-hypoxic state and lactate acidosis [82, 83].

Targeted inhibiting the Warburg effect enhances the radiosensitivity of tumors. Bol et al. found that Warburgphenotype tumor cells with impaired mitochondrial respiration were radiosensitive compared with wild-type parental cells [22]. Pyruvate kinase M2 isoform (PKM2) is a key regulator of the Warburg effect. It is expressed exclusively in cancers. Meng et al. showed that silencing PKM2 expression by shRNA enhanced radiation-induced apoptosis and autophagy in non-small cell lung cancer cell lines and xenografts [21]. Pitroda et al. identified new functions of STAT1, that its knockdown significantly induced radiosensitization of irradiated tumors, which indicated that STAT1 activation enhances the radioresistance of tumors [21]. Furthermore, mutation of tumor protein 53 (TP53) in head and neck squamous cell carcinoma (HNSCC) cells was found to be correlated with the Warburg effect. TP53 mutations were also correlated with HNSCC radioresistance. Sandulache et al. reported that the TP53 mutational status may be used as a marker to overcome radioresistance [29]. In addition, Pena-Rico et al. found that knockdown of TP53 induced the expression of a glycolysis and apoptosis regulator (TIGAR), resulting in radiosensitization of glioma cells [23]. The inhibition of Glut-1 has also been shown to sensitize radioresistant breast cancer cells to irradiation [14].

\section{The Warburg effect and radioresistance of laryngeal carcinoma}

In a review, we reported that Glut-1 and the phosphatidylinositol 3-kinase/protein kinase B pathway are associated with cancer radioresistance [25]. In the case of laryngeal carcinoma, similar results have also been revealed. Finally, in a previous study, we found that hypoxia enhanced the radioresistance of CD133positive Hep-2 cells [26]. Our previous studies also revealed that inhibition of Glut-1 expression by antisense oligodeoxynucleotides and the PI3K/Akt signaling pathway can improve the radiosensitivity of laryngeal carcinoma [27, 28]. Thus, we think that the Warburg effect may be linked to the radiosensitivity of laryngeal carcinoma.

\section{HK-II AND RADIORESISTANCE}

\section{HK-II and radioresistance of cancer}

HK-II is highly expressed in a variety of cancers. Tian et al. showed that HK-II was overexpressed within oral squamous cell carcinoma by HK-II immunohistochemical staining of tumor sections from 19 patients [32]. Increased HK-II expression was also seen in GBM [84]. Clinically, HK-II expression correlates with worse overall survival of GBM patients [85]. Similar results have also been shown in $\mathrm{HCC}$, in which $\mathrm{HK}$ II overexpression was induced by hypoxia [34, 86]. It was also shown that hypoxia-mediated HK-II induction enhanced mitochondrial stability. The inhibition of HK-II caused the release of this enzyme from the permeability transition pore complex, thus leading to the activation of mitochondrial apoptotic signals [33]. Another study showed that the combination of positive HK-II and negative phosphorylated pyruvate dehydrogenase-E1alpha (p-PDH) was associated with reduced recurrence-free survival of colorectal tumor patients [36]. In addition, for breast cancer, direct inhibition of the enzymatic function of HK-II using metformin caused evident tumor necrosis [35]. These results indicate that HK-II promotes tumor progression.

It has been revealed that HK-II is overexpressed after RT in prostate cancer patients [87]. And the depletion of HK-II increases the sensitivity of GBM cells to radiation [85], which suggested that the high expression of HK-II enhances the radioresistance of tumors.

\section{HK-II and radioresistance of laryngeal carcinoma}

The high expression of HK-II has already been investigated in laryngeal carcinoma. Chen et al. assessed HK-II expression in laryngeal squamous cell carcinoma tissues by immunohistochemistry and found that it was significantly higher in this condition than in papilloma or glottis polypus. Stronger HK-II staining was also found to be associated with higher T, N, and TNM stages. This research group then inhibited HK-II expression in Hep-2 cells using shRNA and found that cells expressing shRNA demonstrated a higher G0:G1 ratio, increased apoptosis, and reduced viability. In addition, xenograft tumors derived from cells expressing shRNA were smaller and less proliferative than those from untransfected or controlplasmid-transfected cells [31]. This study suggests that HK-II promotes the development of laryngeal carcinoma.

Min et al. demonstrated that codepletion of Inositol polyphosphate 4-phosphatase type II (INPP4B) and HK-II notably sensitized radioresistant-Hep-2 cells to irradiation. In addition, HK-II depletion sensitized radioresistantHep-2 cells to radiation, although the result did not reach significance [40]. 


\section{Possible mechanisms of enhancing the radiosensitivity of laryngeal carcinoma via HK-II regulation}

Accumulating evidence has shown that dysregulated signaling pathways often result in HK-II overexpression. Various molecular mechanisms, including genetic and epigenetic mechanisms, oncogenes, tumor suppressor genes, and microRNAs (miRNAs), have been suggested to account for the altered expression of HK-II in cancers.

\section{HIF-1 $\alpha$ is involved in the HK-II-mediated Warburg effect}

HIF- $1 \alpha$ is a subunit of a heterodimeric transcription factor, HIF-1, which is encoded by the HIF1A gene. HIF1ais elevated in many different cancers. It is a major transcriptional regulator of the cellular metabolism response to hypoxia, which is common in the tumor microenvironment. The expression of HIF-1 $\alpha$ was found to be positively correlated with the expression of HK-II in gastric adenocarcinoma [88]. The activation of HIF$1 \alpha$ under hypoxic conditions increases glycolysis by inducing glycolysis-regulatory genes including HK-II [89, 90]. A review has also indicated that HIF-1 enhanced the radioresistance of cancer cells through the reprogramming of glucose metabolism by increasing the expression of glycolytic enzymes, including HK-II [15].

INPP4B is a regulatory enzyme that selectively removes the phosphate from the fourth position of the inositol ring in phosphatidylinositol( 3,4$)$-bisphosphate, which is involved in the phosphatidylinositol signaling pathway [91]. Kim et al. demonstrated that INPP4B is a novel marker of radioresistance of laryngeal carcinoma, which is highly expressed in radioresistant laryngeal carcinoma [47]. In addition to irradiation, hypoxia also induces INPP4B expression. Min et al. found that the level of INPP4B was induced under hypoxic conditions, concomitant with an increase in HIF-1 $\alpha$ protein levels in laryngeal carcinoma Hep-2 cells. After transfection of siRNA against HIF- $1 \alpha$, both hypoxia- and radiationinduced INPP4B expression decreased, indicating that such expression is increased by HIF-1 $\alpha$ in laryngeal carcinoma Hep-2 cells [41]. In addition, the association of INPP4B-mediated radioresistance with Akt survival signaling has been revealed [47]. In another study, silencing of INPP4B blocked the activation of Akt, while the overexpression of INPP4B increased proliferation and triggered the anchorage-independent growth of normal colon epithelial cells [92]. Stress-induced INPP4B expression exerted a cytoprotective effect by suppressing apoptotic cell death via upregulation of Akt activation.

The serine/threonine kinase Akt, also known as protein kinase $\mathrm{B}$, has emerged as one of the most frequently activated protein kinases in human cancer. It has been referred to as a "Warburg kinase" [93]. Shimura et al. found that inhibition of the Akt pathway by API-
2, an Akt inhibitor, eliminated the aerobic glycolysis enhanced by increased Glut-1 expression in HepG2 (human liver cancer) and HeLa (cervical cancer) cell lines, which exhibits radioresistance acquired by longterm exposure to fractionated radiation. They also found that inhibiting glycolysis using 2-DG, an HK-II inhibitor, suppressed acquired tumor cell radioresistance [17]. In fact, the activity of HK-II is thought to be induced by Akt. Akt activity promotes both the binding of HK-II to mitochondria and glucose uptake in cancer cells [94]. The mechanisms by which Akt induces the activity of HKII have not been fully elucidated. However, in a recent review, Roberts and Miyamoto described that HK-II expression was upregulated by the Akt/mechanistic target of rapamycin complex 1 (mTORC1) pathway [95].

The Akt-mechanistic target of rapamycin (mTOR) pathway is strongly associated with increased glycolysis in tumors [96]. mTOR is considered an upstream activator of HIF-1 $\alpha$, EIF4E-binding protein 1 (4E-BP1), and S6 kinase (S6K), which are upstream activators of HK-II in the Warburg effect in cancer cells [40, 97]. mTOR is also a suppressor of some miRNAs, such as miR-143 and miR-125b, which are upstream suppressors of HK-II in the Warburg effect [98, 99]. In HNSCC-derived cell lines, miR-143 expression was inversely correlated with HK-II level. Furthermore, miR-143 was shown to inhibit HKII expression via a conserved miR-143 recognition motif located in the 3'-untranslated region of HK-II mRNA [100]. This mechanism has also been demonstrated in lung cancer, colon cancer, and renal cell carcinoma [77, 98, 101]. In addition to miR-143, miR-125b targeted inhibits HK-II to sensitize human HCC cells to chemotherapy [99].

\section{The role of suppressor oncogenes in the HK-II- mediated Warburg effect}

Apart from HIF-1 $\alpha$, other signaling pathways have been proposed to be involved in the HK-II-mediated Warburg effect. Deficiencies in the tumor suppressor genes phosphatase and tensin homologue (PTEN) and p53 were found in the HK-II-mediated Warburg effect.

miRNA-21 (miR-21)/PTEN/PI3K/Akt/mTOR signaling pathway

The PTEN/Akt/mTOR pathway was the focus of a review by Steelman et al. It was shown to promote the growth and suppress the sensitivity of cancer to therapy [97]. PTEN is frequently inactivated in many human cancers via point mutations, among others. The deficiency or inactivation of PTEN leads to elevated Akt activity and unrestricted proliferation. The involvement of the PTEN/Akt/mTOR/4E-BP1 pathway in the HK-IImediated Warburg effect was subsequently confirmed by Wang et al. in prostate cancer. HK-II protein expression is increased by PTEN deletion. This research group subsequently depleted or knocked down Akt or Raptor (an essential component of the mTORC1 complex) in PTEN/ p53 double-deficient MEFs or the prostate cancer cell line 
UMN-4240P, resulting in a significant reduction in HK-II protein expression. In a later study, the pharmacological inhibition of Akt/mTORC1 signaling by NVP-BEZ235 was found to reduce HK-II protein expression remarkably. As BEZ235 significantly inhibited the activation of 4E$\mathrm{BP} 1$, which is the primary downstream effector in the AktmTORC1 pathway, it was suggested that 4E-BP1 might increase HK-II expression [102].

Recently, it was demonstrated that inhibition of miR-21 in bladder cancer cell lines reduced glucose uptake, lactate production, and HK activity, which are considered to be related to the Warburg effect [103, 104]. Meanwhile, miR-21 inhibition promoted PTEN expression, decreased phosphorylated Akt, and deactivated mTOR [105]. Other studies identified PTEN as a target gene of miR-21 in colorectal cancer, non-small cell lung cancer, intrahepatic cholangiocarcinoma, ovarian cancer, cervical cancer, esophageal cancer, and HER2-positive gastric cancer [106-114]. It was also revealed that miR-21 expression was upregulated in laryngeal carcinoma, while PTEN expression was downregulated. The level of miR21 was also found to be inversely correlated with PTEN expression [115].

PTEN has actually been reported to be an antagonist of PI3K signaling, which mediates the Akt/mTOR pathway. Hopkins et al. showed that a 576-amino-acid translational variant of PTEN (named PTEN-Long), which is a membrane-permeable lipid phosphatase secreted from cells that can enter other cells, antagonized PI3K signaling and induced tumor cell death in vitro and in vivo [116]. $\mathrm{PI} 3 \mathrm{~K}$ is a heterodimeric protein with an $85-\mathrm{kDa}$ regulatory subunit and a $110-\mathrm{kDa}$ catalytic subunit (PIK3CA). In an earlier study, the regulation of Akt by lipid products of PI3K was investigated by Franke et al. They found that Akt activity was correlated with the amount of phosphatidylinositol-3,4-bisphosphate (PtdIns-3,4-P2) in vivo, and synthetic PtdIns-3,4-P2 activated Akt both in vitro and in vivo. Akt is apparently activated by the direct correlation of PtdIns-3,4-P2 with the Akt PH domain [117]. Subsequently, Ahn et al. established SNU449 cells transfected with the HK-II gene using an expression vector. They found that the activated form of Akt was increased after transfection, and the PI3K inhibitor led to dissociation of mitochondrial HK-II into the cytoplasm. This revealed that HK-II promotes (18)F-FDG uptake and tumor proliferation via $\mathrm{PI} 3 \mathrm{~K}$-dependent Akt signaling pathways [118]. The PI3K/Akt/mTOR signaling pathway was also reported to be involved in leukemogenesis [119]. Recently, it was reported that elevated HK-II expression was induced by activated PI3K/Akt signaling in osteosarcoma. Liu et al. found that the inhibition of $\mathrm{PI} 3 \mathrm{~K} / \mathrm{Akt}$ signaling suppressed aerobic glycolysis, which could be reversed by reintroduction of HK-II [120].

\section{p53/miR-143 pathway}

Similarly to PTEN, another tumor suppressor gene, p53, was also found to be involved in the HK-II-mediated
Warburg effect [90]. HK-II was selectively upregulated by the combined loss of PTEN and p53 in prostate cancer cells. In fact, p53 loss enhances HK-II mRNA stability through the inhibition of miR143 biogenesis [102], which, as discussed above, inhibits HK-II expression in various cancer cells [98]. It has also been revealed that the suppression of $\mathrm{p} 53$ activation by aldehyde reductase (AKR1A1) results in the acquisition of radioresistance by laryngeal carcinoma [46].

c-MYC-mediated high expression of HK-II

The oncogene c-MYC was positively correlated with the expression of HK-II. In fact, c-MYC regulates the transcription of genes involved in nearly every step of the glycolytic pathway, including HK-II, and enhances aerobic glycolysis [90]. Alvarez et al. demonstrated that c-MYCdriven tumors were characterized by high expression of HK-II and exhibited both high FDG uptake and rapid proliferation [121].

\section{miR-155 upregulates HK-II through two different signaling pathways}

miRNAs are now considered regulators involved in the HK-II-mediated Warburg effect. A recent study revealed that the miR-155/HK-II axis is an important regulator of tumor plasticity and may be useful for predicting the response and adaptation to aromatase inhibitors in breast cancer patients [122]. According to another study by Jiang et al., miR-155, which is thought to be a pro-inflammatory cytokine, upregulates HK-II through the miR-155/suppressor of cytokine signaling 1 (SOCS1)/STAT3 and miR-155/CCAAT enhancer binding protein $\beta(\mathrm{C} / \mathrm{EBP} \beta) / \mathrm{miR}-143$ signaling pathways [123].

miR-155/SOCS1/STAT3 signaling pathway

The Warburg effect was strongly increased by mir155 overexpression and significantly decreased by its knockdown. The HK-II protein level was also dramatically increased by miR-155 and reduced by anti-miR-155. These results suggest that mir-155 increases the Warburg effect by increasing HK-II protein expression.

STAT3 signaling is a major pathway that connects inflammation to cancer. It has been revealed that the long non-coding RNAs (lncRNAs) urothelial cancer-associated 1 (UCA1) promotes glycolysis in bladder cancer cells via the mTOR/STAT3/HK-II cascade [124]. In addition, in a recent study, Choe et al. demonstrated an increased correlation between ERp57 and STAT3 in radioresistant laryngeal carcinoma. Furthermore, the inhibition of STAT3 activity using a chemical inhibitor sensitized radioresistant cells to irradiation. These results suggest that the ERp57/ STAT3 axis increases the radioresistance of laryngeal carcinoma. This research group also found that an increase in the level of the ERp57-STAT3 complex was associated with a poor prognosis in human laryngeal carcinoma [44].

To explore whether STAT3 directly upregulates HK-II, Jiang et al. used siRNA to knock down STAT3, which caused a significant reduction in the level of HK- 
II mRNA. Meanwhile, STAT3 inhibition by the addition of JSI-124 (a STAT3 inhibitor) significantly reduced the activity of HK-II. The finding that miR-155 contributes to STAT3 activation via suppression of SOCS1 indicates that the miR-155/SOCS1/STAT3 signaling pathway is involved in the Warburg effect [123].

miR-155/(C/EBP $\beta) / m i R-143$ signaling pathway

miR-143 directly suppresses HK-II expression in various cancer cells $[77,98,100,101]$. According to a study by Jiang et al., mir-143 expression was inversely correlated with mir-155 expression in breast cancer cell lines. To explore the mechanisms underlying miR-155induced activity of miR-143, C/EBP $\beta$ (a miR-155 target) was knocked down, which strongly reduced miR-143 expression in ZR-75-30 cells, while the overexpression of $\mathrm{C} / \mathrm{EBP} \beta$ led to an increase in the miR-143 level. In addition, the transfection of miR-155 into ZR-75-30 cells significantly decreased miR-143 expression and C/EBP $\beta$ protein levels. This miR-143 inhibition was rescued upon co-expression of a miR-155-resistant form of $\mathrm{C} / \mathrm{EBP} \beta$ [123]. All of these results indicate that the miR-155/(C/ $\mathrm{EBP} \beta) / \mathrm{miR}-143$ signaling pathway is involved in the Warburg effect.

Besides the signaling pathways stated above, there are also other regulators involved in the Warburg effect. SLUG (also known as Snai2) is a zinc-finger transcription factor correlated with poor outcomes in various cancers. In a recent study, Geng et al. demonstrated that curcumin (a major component of the food flavoring turmeric and an anticarcinogenic agent) promoted 4-hydroxytamoxifen (4-OHT) sensitivity of triple-negative breast cancer (lacking ER, PR, and HER2) by inhibiting the SLUG/HKII signaling pathway [125]. Furthermore, in a study on CA-IX, an enzyme that lowers $\mathrm{pH}$, Yu et al. revealed that the inhibition of hypoxia-induced CA-IX enhanced HK-II inhibitor-induced apoptosis of HCC cells [60].

Although the exact mechanisms underlying HK-IImediated radioresistance of laryngeal carcinoma have not been fully elucidated, as a number of factors regulating HK-II expression have already been investigated, we speculate that novel inhibitors targeting the HK-IImediated Warburg effect may emerge as promising tools to enhance the radiosensitivity of laryngeal carcinoma.

\section{CONCLUSIONS AND THERAPEUTIC PERSPECTIVES}

Although RT has been used as a nonsurgical strategy for organ preservation in the appropriate patients with advanced laryngeal carcinoma, resistance to RT remains a major problem, especially for advanced-stage tumors. The Warburg effect has been considered one of the major causes of radioresistance in all kinds of tumor cells, including laryngeal carcinoma. HK-II, which is a key enzyme in the Warburg effect and is positively correlated with the survival of various tumor cells, is highly expressed in laryngeal carcinoma. Numerous agents (e.g., 2-DG, lonidamine, 3-BP, metformin, MJ, oroxylin A, casiopeina II-gly, NA, prosapogenin A, clotrimazole, bifonazole, and anti-HK-II shRNA) have already been revealed to be useful anticancer therapies by targeted inhibiting HK-II or the HK-II-VDAC complex. Thus, we speculate that agents targeted inhibiting HK-II may attenuate the Warburg effect in laryngeal carcinoma and sensitize the cancer cells to RT. The HIF-1 $\alpha /$ INPP4B/ Akt/mTOR/S6K, PTEN/Akt/mTOR/4E-BP1, miR-155/ SOCS1/STAT3, miR-155/(C/EBP $\beta) / \mathrm{miR}-143$, and p53/ miR-143 signaling pathways, as well as c-Myc and CAIX, may be involved in the mechanisms elevating HK-II expression in laryngeal carcinoma. Although some studies have indicated that targeted inhibiting the HK-II-VDAC complex may be a promising approach for anticancer therapies, no effective agents have yet reached the clinical stage. Further studies and clinical trials on effective agents targeted inhibiting the signaling pathways of HK-II in laryngeal carcinoma are thus still required. Nonetheless, the findings obtained thus far indicate that targeted inhibiting HK-II may be a novel approach to enhance the radiosensitivity of laryngeal carcinoma.

\section{ACKNOWLEDGMENTS}

This research was supported by National Natural Science Foundation of China (No. 81172562 and 81372903), and Science and Technology Department of Zhejiang Province, China (No.2016C33144).

\section{CONFLICTS OF INTEREST}

None.

\section{REFERENCES}

1. www.cancerresearchuk.org.

2. Rosenberg PJ. Total laryngectomy and cancer of the larynx. A historical review. Archives of otolaryngology. 1971; 94:313-316.

3. Tomeh $\mathrm{C}$ and Holsinger FC. Laryngeal cancer. Current opinion in otolaryngology \& head and neck surgery. 2014; 22:147-153.

4. Denaro N, Russi EG, Lefebvre JL and Merlano MC. A systematic review of current and emerging approaches in the field of larynx preservation. Radiotherapy and oncology. 2014; 110:16-24.

5. Nguyen NP, Chi A, Betz M, Almeida F, Vos P, Davis R, Slane B, Ceizyk M, Abraham D, Smith-Raymond L, Stevie M, Jang S, Gelumbauskas S, et al. Feasibility of intensitymodulated and image-guided radiotherapy for functional organ preservation in locally advanced laryngeal cancer. PloS one. 2012; 7:e42729.

6. Nutting CM, Morden JP, Harrington KJ, Urbano TG, Bhide 
SA, Clark C, Miles EA, Miah AB, Newbold K, Tanay M, Adab F, Jefferies SJ, Scrase C, et al. Parotid-sparing intensity modulated versus conventional radiotherapy in head and neck cancer (PARSPORT): a phase 3 multicentre randomised controlled trial. The Lancet Oncology. 2011; 12:127-136.

7. Bhide SA, Newbold KL, Harrington KJ and Nutting CM. Clinical evaluation of intensity-modulated radiotherapy for head and neck cancers. The British journal of radiology. 2012; 85:487-494.

8. Grotenhuis BA, Wijnhoven BP and van Lanschot JJ. Cancer stem cells and their potential implications for the treatment of solid tumors. Journal of surgical oncology. 2012; 106:209-215.

9. Diehn M, Cho RW, Lobo NA, Kalisky T, Dorie MJ, Kulp AN, Qian D, Lam JS, Ailles LE, Wong M, Joshua B, Kaplan MJ, Wapnir I, et al. Association of reactive oxygen species levels and radioresistance in cancer stem cells. Nature. 2009; 458:780-783.

10. D'Andrea FP. Intrinsic radiation resistance of mesenchymal cancer stem cells and implications for treatment response in a murine sarcoma model. Danish medical journal. 2012; 59:B4388.

11. Krause M, Yaromina A, Eicheler W, Koch U and Baumann M. Cancer stem cells: targets and potential biomarkers for radiotherapy. Clinical cancer research. 2011; 17:7224-7229.

12. Milosevic M, Warde P, Menard C, Chung P, Toi A, Ishkanian A, McLean M, Pintilie M, Sykes J, Gospodarowicz M, Catton C, Hill RP and Bristow R. Tumor hypoxia predicts biochemical failure following radiotherapy for clinically localized prostate cancer. Clinical cancer research. 2012; 18:2108-2114.

13. Spanos WC, Nowicki P, Lee DW, Hoover A, Hostager B, Gupta A, Anderson ME and Lee JH. Immune response during therapy with cisplatin or radiation for human papillomavirus-related head and neck cancer. Archives of otolaryngology—head \& neck surgery. 2009; 135:11371146.

14. Zhao F, Ming J, Zhou Y and Fan L. Inhibition of Glut by WZB117 sensitizes radioresistant breast cancer cells to irradiation. Cancer chemotherapy and pharmacology. 2016.

15. Harada H. Hypoxia-inducible factor 1-mediated characteristic features of cancer cells for tumor radioresistance. Journal of radiation research. 2016.

16. Bhatt AN, Chauhan A, Khanna S, Rai Y, Singh S, Soni $\mathrm{R}$, Kalra N and Dwarakanath BS. Transient elevation of glycolysis confers radio-resistance by facilitating DNA repair in cells. BMC cancer. 2015; 15:335.

17. Shimura T, Noma N, Sano Y, Ochiai Y, Oikawa T, Fukumoto M and Kunugita N. AKT-mediated enhanced aerobic glycolysis causes acquired radioresistance by human tumor cells. Radiotherapy and oncology. 2014; 112:302-307.

18. Koukourakis MI, Giatromanolaki A, Panteliadou M,
Pouliliou SE, Chondrou PS, Mavropoulou S and Sivridis E. Lactate dehydrogenase 5 isoenzyme overexpression defines resistance of prostate cancer to radiotherapy. British journal of cancer. 2014; 110:2217-2223.

19. Hirschhaeuser F, Sattler UG and Mueller-Klieser W. Lactate: a metabolic key player in cancer. Cancer research. 2011; 71:6921-6925.

20. Pitroda SP, Wakim BT, Sood RF, Beveridge MG, Beckett MA, MacDermed DM, Weichselbaum RR and Khodarev NN. STAT1-dependent expression of energy metabolic pathways links tumour growth and radioresistance to the Warburg effect. BMC medicine. 2009; 7:68.

21. Meng MB, Wang HH, Guo WH, Wu ZQ, Zeng XL, Zaorsky NG, Shi HS, Qian D, Niu ZM, Jiang B, Zhao LJ, Yuan ZY and Wang P. Targeting pyruvate kinase M2 contributes to radiosensitivity of non-small cell lung cancer cells in vitro and in vivo. Cancer letters. 2015; 356:985-993.

22. Bol V, Bol A, Bouzin C, Labar D, Lee JA, Janssens G, Porporato PE, Sonveaux P, Feron O and Gregoire V. Reprogramming of tumor metabolism by targeting mitochondria improves tumor response to irradiation. Acta oncologica (Stockholm, Sweden). 2015; 54:266-274.

23. Pena-Rico MA, Calvo-Vidal MN, Villalonga-Planells R, Martinez-Soler F, Gimenez-Bonafe P, Navarro-Sabate A, Tortosa A, Bartrons R and Manzano A. TP53 induced glycolysis and apoptosis regulator (TIGAR) knockdown results in radiosensitization of glioma cells. Radiotherapy and oncology. 2011; 101:132-139.

24. Mims J, Bansal N, Bharadwaj MS, Chen X, Molina AJ, Tsang AW and Furdui CM. Energy metabolism in a matched model of radiation resistance for head and neck squamous cell cancer. Radiation research. 2015; 183:291304.

25. Fang J, Zhou SH, Fan J and Yan SX. Roles of glucose transporter-1 and the phosphatidylinositol 3kinase/protein kinase B pathway in cancer radioresistance (review). Molecular medicine reports. 2015; 11:1573-1581.

26. Wang M, Li X, Qu Y, Xu O and Sun Q. Hypoxia promotes radioresistance of CD133-positive Hep-2 human laryngeal squamous carcinoma cells in vitro. International journal of oncology. 2013; 43:131-140.

27. Luo XM, Xu B, Zhou ML, Bao YY, Zhou SH, Fan J and Lu ZJ. Co-Inhibition of GLUT-1 Expression and the PI3K/ Akt Signaling Pathway to Enhance the Radiosensitivity of Laryngeal Carcinoma Xenografts In Vivo. PloS one. 2015; 10:e0143306.

28. Yan SX, Luo XM, Zhou SH, Bao YY, Fan J, Lu ZJ, Liao XB, Huang YP, Wu TT and Wang QY. Effect of antisense oligodeoxynucleotides glucose transporter-1 on enhancement of radiosensitivity of laryngeal carcinoma. International journal of medical sciences. 2013; 10:13751386.

29. Sandulache VC, Skinner HD, Ow TJ, Zhang A, Xia X, Luchak JM, Wong LJ, Pickering CR, Zhou G and Myers 
$\mathrm{JN}$. Individualizing antimetabolic treatment strategies for head and neck squamous cell carcinoma based on TP53 mutational status. Cancer. 2012; 118:711-721.

30. Mathupala SP, Ko YH and Pedersen PL. Hexokinase-2 bound to mitochondria: cancer's stygian link to the "Warburg Effect" and a pivotal target for effective therapy. Seminars in cancer biology. 2009; 19:17-24.

31. Chen J, Zhang S, Li Y, Tang Z and Kong W. Hexokinase 2 overexpression promotes the proliferation and survival of laryngeal squamous cell carcinoma. Tumour biology. 2014; 35:3743-3753.

32. Tian M, Zhang H, Higuchi T, Oriuchi N, Nakasone Y, Takata K, Nakajima N, Mogi K and Endo K. HexokinaseII expression in untreated oral squamous cell carcinoma: comparison with FDG PET imaging. Annals of nuclear medicine. 2005; 19:335-338.

33. Kim W, Yoon JH, Jeong JM, Cheon GJ, Lee TS, Yang JI, Park SC and Lee HS. Apoptosis-inducing antitumor efficacy of hexokinase II inhibitor in hepatocellular carcinoma. Molecular cancer therapeutics. 2007; 6:2554-2562.

34. Guzman G, Chennuri R, Chan A, Rea B, Quintana A, Patel $\mathrm{R}, \mathrm{Xu} \mathrm{PZ}, \mathrm{Xie} \mathrm{H}$ and Hay N. Evidence for heightened hexokinase II immunoexpression in hepatocyte dysplasia and hepatocellular carcinoma. Digestive diseases and sciences. 2015; 60:420-426.

35. Marini C, Salani B, Massollo M, Amaro A, Esposito AI, Orengo AM, Capitanio S, Emionite L, Riondato M, Bottoni G, Massara C, Boccardo S, Fabbi M, et al. Direct inhibition of hexokinase activity by metformin at least partially impairs glucose metabolism and tumor growth in experimental breast cancer. Cell cycle. 2013; 12:3490-3499.

36. Hamabe A, Yamamoto H, Konno M, Uemura M, Nishimura J, Hata T, Takemasa I, Mizushima T, Nishida N, Kawamoto K, Koseki J, Doki Y, Mori M, et al. Combined evaluation of hexokinase 2 and phosphorylated pyruvate dehydrogenaseE1alpha in invasive front lesions of colorectal tumors predicts cancer metabolism and patient prognosis. Cancer science. 2014; 105:1100-1108.

37. Patra KC, Wang Q, Bhaskar PT, Miller L, Wang Z, Wheaton W, Chandel N, Laakso M, Muller WJ, Allen EL, Jha AK, Smolen GA, Clasquin MF, et al. Hexokinase 2 is required for tumor initiation and maintenance and its systemic deletion is therapeutic in mouse models of cancer. Cancer cell. 2013; 24:213-228.

38. Krasnov GS, Dmitriev AA, Lakunina VA, Kirpiy AA and Kudryavtseva AV. Targeting VDAC-bound hexokinase II: a promising approach for concomitant anti-cancer therapy. Expert opinion on therapeutic targets. 2013; 17:1221-1233.

39. Cerella C, Dicato $M$ and Diederich M. Modulatory roles of glycolytic enzymes in cell death. Biochemical pharmacology. 2014; 92:22-30.

40. Min JW, Kim KI, Kim HA, Kim EK, Noh WC, Jeon HB, Cho DH, Oh JS, Park IC, Hwang SG and Kim JS. INPP4Bmediated tumor resistance is associated with modulation of glucose metabolism via hexokinase 2 regulation in laryngeal cancer cells. Biochemical and biophysical research communications. 2013; 440:137-142.

41. Zamulaeva IA, Matchuk ON, Selivanova EI, Andreev VG, Lipunov NM, Makarenko SA, Zhavoronkov LP and Saenko AS. [Increase in the number of cancer stem cells after exposure to low-LET radiation]. Radiatsionnaia biologiia, radioecologiia / Rossiiskaia akademiia nauk. 2014; 54:256264.

42. Wang M, Li X, Lu X, Qu Y, Xu O and Sun Q. [Cancer stem cells promotes resistance of laryngeal squamous cancer to irradiation mediated by hypoxia]. Lin Chung Er Bi Yan Hou Tou Jing Wai Ke Za Zhi. 2011; 25:823-826.

43. Maia D, de Carvalho AC, Horst MA, Carvalho AL, Scapulatempo-Neto C and Vettore AL. Expression of miR296-5p as predictive marker for radiotherapy resistance in early-stage laryngeal carcinoma. Journal of translational medicine. 2015; 13:262.

44. Choe MH, Min JW, Jeon HB, Cho DH, Oh JS, Lee HG, Hwang SG, An S, Han YH and Kim JS. ERp57 modulates STAT3 activity in radioresistant laryngeal cancer cells and serves as a prognostic marker for laryngeal cancer. Oncotarget. 2015; 6:2654-2666. doi: 10.18632/ oncotarget.3042.

45. Murakami N, Mori T, Yoshimoto S, Ito Y, Kobayashi K, Ken H, Kitaguchi M, Sekii S, Takahashi K, Yoshio K, Inaba K, Morota M, Sumi M, et al. Expression of EpCAM and prognosis in early-stage glottic cancer treated by radiotherapy. The Laryngoscope. 2014; 124:E431-436.

46. Kim JS, Chang JW, Park JK and Hwang SG. Increased aldehyde reductase expression mediates acquired radioresistance of laryngeal cancer cells via modulating p53. Cancer biology \& therapy. 2012; 13:638-646.

47. Kim JS, Yun HS, Um HD, Park JK, Lee KH, Kang CM, Lee SJ and Hwang SG. Identification of inositol polyphosphate 4-phosphatase type II as a novel tumor resistance biomarker in human laryngeal cancer HEp-2 cells. Cancer biology \& therapy. 2012; 13:1307-1318.

48. Nijkamp MM, Span PN, Terhaard CH, Doornaert PA, Langendijk JA, van den Ende PL, de Jong M, van der Kogel AJ, Bussink J and Kaanders JH. Epidermal growth factor receptor expression in laryngeal cancer predicts the effect of hypoxia modification as an additive to accelerated radiotherapy in a randomised controlled trial. Eur J Cancer. 2013; 49:3202-3209.

49. Nichols AC, Whelan F, Basmaji J, Dhaliwal S, Dowthwaite S, Chapeskie C, Read N, Palma DA, Fung K, Venkatesan V, Hammond JA, Franklin JH, Siddiqui I, et al. Ki-67 expression predicts radiotherapy failure in early glottic cancer. Journal of otolaryngology - head \& neck surgery. 2012; 41:124-130.

50. Gioacchini FM, Alicandri-Ciufelli M, Rubini C, Magliulo $\mathrm{G}$ and $\mathrm{Re} \mathrm{M}$. Prognostic value of $\mathrm{Bcl}-2$ expression in squamous cell carcinoma of the larynx: a systematic review. The International journal of biological markers. 2015; 
30:e155-160.

51. Chen XH, Bao YY, Zhou SH, Wang QY, Wei Y and Fan J. Glucose transporter-1 expression in CD133+ laryngeal carcinoma Hep-2 cells. Molecular medicine reports. 2013; 8:1695-1700.

52. Kim JS, Kim SY, Lee M, Kim SH, Kim SM and Kim EJ. Radioresistance in a human laryngeal squamous cell carcinoma cell line is associated with DNA methylation changes and topoisomerase II alpha. Cancer biology \& therapy. 2015; 16:558-566.

53. Ke Q, Wu J, Ming B, Zhu S, Yu M, Wang Y, Hu C, Xu S and Luo Z. Identification of the PAG1 gene as a novel target of inherent radioresistance in human laryngeal carcinoma cells. Cancer biotherapy \& radiopharmaceuticals. 2012; 27:678-684.

54. Yang B, Tang F, Zhang B, Zhao Y, Ding S and Rao Z. Comparative ultrastructure analysis of radiationinduced radioresistant laryngeal cancer hep-2 cell line. Ultrastructural pathology. 2014; 38:285-289.

55. Mohanti BK, Rath GK, Anantha N, Kannan V, Das BS, Chandramouli BA, Banerjee AK, Das S, Jena A, Ravichandran R, Sahi UP, Kumar R, Kapoor N, et al. Improving cancer radiotherapy with 2-deoxy-D-glucose: phase I/II clinical trials on human cerebral gliomas. International journal of radiation oncology, biology, physics. 1996; 35:103-111.

56. Stein M, Lin H, Jeyamohan C, Dvorzhinski D, Gounder M, Bray K, Eddy S, Goodin S, White E and Dipaola RS. Targeting tumor metabolism with 2-deoxyglucose in patients with castrate-resistant prostate cancer and advanced malignancies. The Prostate. 2010; 70:1388-1394.

57. Wang J, Jiang Z, Xiang L, Li Y, Ou M, Yang X, Shao J, Lu Y, Lin L, Chen J, Dai Y and Jia L. Synergism of ursolic acid derivative US597 with 2-deoxy-D-glucose to preferentially induce tumor cell death by dual-targeting of apoptosis and glycolysis. Scientific reports. 2014; 4:5006.

58. Wang Z, Zhang L, Zhang D, Sun R, Wang Q and Liu $\mathrm{X}$. Glycolysis inhibitor 2-deoxy-D-glucose suppresses carcinogen-induced rat hepatocarcinogenesis by restricting cancer cell metabolism. Molecular medicine reports. 2015; 11:1917-1924.

59. Buijs M, Vossen JA, Geschwind JF, Ishimori T, Engles JM, Acha-Ngwodo O, Wahl RL and Vali M. Specificity of the anti-glycolytic activity of 3-bromopyruvate confirmed by FDG uptake in a rat model of breast cancer. Investigational new drugs. 2009; 27:120-123.

60. Yu SJ, Yoon JH, Lee JH, Myung SJ, Jang ES, Kwak MS, Cho EJ, Jang JJ, Kim YJ and Lee HS. Inhibition of hypoxiainducible carbonic anhydrase-IX enhances hexokinase II inhibitor-induced hepatocellular carcinoma cell apoptosis. Acta pharmacologica Sinica. 2011; 32:912-920.

61. Yu SJ, Yoon JH, Yang JI, Cho EJ, Kwak MS, Jang ES, Lee JH, Kim YJ, Lee HS and Kim CY. Enhancement of hexokinase II inhibitor-induced apoptosis in hepatocellular carcinoma cells via augmenting ER stress and antiangiogenesis by protein disulfide isomerase inhibition. Journal of bioenergetics and biomembranes. 2012; 44:101115.

62. Gong L, Wei Y, Yu X, Peng J and Leng X. 3-Bromopyruvic acid, a hexokinase II inhibitor, is an effective antitumor agent on the hepatoma cells : in vitro and in vivo findings. Anti-cancer agents in medicinal chemistry. 2014; 14:771776.

63. Nakano A, Miki H, Nakamura S, Harada T, Oda A, Amou H, Fujii S, Kagawa K, Takeuchi K, Ozaki S, Matsumoto $\mathrm{T}$ and Abe M. Up-regulation of hexokinaseII in myeloma cells: targeting myeloma cells with 3-bromopyruvate. Journal of bioenergetics and biomembranes. 2012; 44:3138.

64. Byrne FL, Poon IK, Modesitt SC, Tomsig JL, Chow JD, Healy ME, Baker WD, Atkins KA, Lancaster JM, Marchion DC, Moley KH, Ravichandran KS, Slack-Davis JK, et al. Metabolic vulnerabilities in endometrial cancer. Cancer research. 2014; 74:5832-5845.

65. Liu Z, Zhang YY, Zhang QW, Zhao SR, Wu CZ, Cheng X, Jiang CC, Jiang ZW and Liu H. 3-Bromopyruvate induces apoptosis in breast cancer cells by downregulating Mcl1 through the PI3K/Akt signaling pathway. Anti-cancer drugs. 2014; 25:447-455.

66. Gandham SK, Talekar M, Singh A and Amiji MM. Inhibition of hexokinase-2 with targeted liposomal 3-bromopyruvate in an ovarian tumor spheroid model of aerobic glycolysis. International journal of nanomedicine. 2015; 10:4405-4423.

67. Galina A. Mitochondria: 3-bromopyruvate vs. mitochondria? A small molecule that attacks tumors by targeting their bioenergetic diversity. The international journal of biochemistry \& cell biology. 2014; 54:266-271.

68. Shuch B, Linehan WM and Srinivasan R. Aerobic glycolysis: a novel target in kidney cancer. Expert review of anticancer therapy. 2013; 13:711-719.

69. Oudard S, Carpentier A, Banu E, Fauchon F, Celerier D, Poupon MF, Dutrillaux B, Andrieu JM and Delattre JY. Phase II study of lonidamine and diazepam in the treatment of recurrent glioblastoma multiforme. Journal of neurooncology. 2003; 63:81-86.

70. Shoshan-Barmatz V and Golan M. Mitochondrial VDAC1: function in cell life and death and a target for cancer therapy. Current medicinal chemistry. 2012; 19:714-735.

71. Mathupala SP, Ko YH and Pedersen PL. Hexokinase II: cancer's double-edged sword acting as both facilitator and gatekeeper of malignancy when bound to mitochondria. Oncogene. 2006; 25:4777-4786.

72. Ramsay EE, Hogg PJ and Dilda PJ. Mitochondrial metabolism inhibitors for cancer therapy. Pharmaceutical research. 2011; 28:2731-2744.

73. Gao JL and Chen YG. Natural compounds regulate glycolysis in hypoxic tumor microenvironment. BioMed 
research international. 2015; 2015:354143.

74. Liu H, Li Y and Raisch KP. Clotrimazole induces a late G1 cell cycle arrest and sensitizes glioblastoma cells to radiation in vitro. Anti-cancer drugs. 2010; 21:841-849.

75. Salani B, Marini C, Rio AD, Ravera S, Massollo M, Orengo AM, Amaro A, Passalacqua M, Maffioli S, Pfeffer U, Cordera R, Maggi D and Sambuceti G. Metformin impairs glucose consumption and survival in Calu-1 cells by direct inhibition of hexokinase-II. Scientific reports. 2013; 3:2070.

76. Hu JW, Sun P, Zhang DX, Xiong WJ and Mi J. Hexokinase 2 regulates G1/S checkpoint through CDK2 in cancerassociated fibroblasts. Cellular signalling. 2014; 26:22102216.

77. Yoshino H, Enokida H, Itesako T, Kojima S, Kinoshita T, Tatarano S, Chiyomaru T, Nakagawa M and Seki N. Tumor-suppressive microRNA-143/145 cluster targets hexokinase-2 in renal cell carcinoma. Cancer science. 2013; 104:1567-1574.

78. Peng Q, Zhou Q, Zhou J, Zhong D, Pan F and Liang H. Stable RNA interference of hexokinase II gene inhibits human colon cancer LoVo cell growth in vitro and in vivo. Cancer biology \& therapy. 2008; 7:1128-1135.

79. Peng Q, Zhou J, Zhou Q, Pan F, Zhong D and Liang H. Silencing hexokinase II gene sensitizes human colon cancer cells to 5-fluorouracil. Hepato-gastroenterology. 2009; 56:355-360.

80. Sullivan EJ, Kurtoglu M, Brenneman R, Liu H and Lampidis TJ. Targeting cisplatin-resistant human tumor cells with metabolic inhibitors. Cancer chemotherapy and pharmacology. 2014; 73:417-427.

81. Kim JE, Ahn BC, Hwang MH, Jeon YH, Jeong SY, Lee SW and Lee J. Combined RNA interference of hexokinase II and (131)I-sodium iodide symporter gene therapy for anaplastic thyroid carcinoma. Journal of nuclear medicine : official publication, Society of Nuclear Medicine. 2011; 52:1756-1763.

82. Leontieva OV and Blagosklonny MV. M(o)TOR of pseudohypoxic state in aging: rapamycin to the rescue. Cell cycle. 2014; 13:509-515.

83. Leontieva OV and Blagosklonny MV. Yeast-like chronological senescence in mammalian cells: phenomenon, mechanism and pharmacological suppression. Aging (Albany NY). 2011; 3:1078-1091. doi: 10.18632/ aging.100402.

84. Wolf A, Agnihotri S, Munoz D and Guha A. Developmental profile and regulation of the glycolytic enzyme hexokinase 2 in normal brain and glioblastoma multiforme. Neurobiology of disease. 2011 ; 44:84-91.

85. Wolf A, Agnihotri S, Micallef J, Mukherjee J, Sabha N, Cairns R, Hawkins C and Guha A. Hexokinase 2 is a key mediator of aerobic glycolysis and promotes tumor growth in human glioblastoma multiforme. The Journal of experimental medicine. 2011; 208:313-326.

86. Gwak GY, Yoon JH, Kim KM, Lee HS, Chung JW and
Gores GJ. Hypoxia stimulates proliferation of human hepatoma cells through the induction of hexokinase II expression. Journal of hepatology. 2005; 42:358-364.

87. Spratt DE, Evans MJ, Davis BJ, Doran MG, Lee MX, Shah N, Wongvipat J, Carnazza KE, Klee GG, Polkinghorn W, Tindall DJ, Lewis JS and Sawyers CL. Androgen Receptor Upregulation Mediates Radioresistance after Ionizing Radiation. Cancer research. 2015; 75:4688-4696.

88. Qiu MZ, Han B, Luo HY, Zhou ZW, Wang ZQ, Wang $\mathrm{FH}, \mathrm{Li} \mathrm{YH}$ and $\mathrm{Xu} \mathrm{RH}$. Expressions of hypoxia-inducible factor-1alpha and hexokinase-II in gastric adenocarcinoma: the impact on prognosis and correlation to clinicopathologic features. Tumour biology. 2011; 32:159-166.

89. Semenza GL. Targeting HIF-1 for cancer therapy. Nature reviews Cancer. 2003; 3:721-732.

90. Yeung SJ, Pan J and Lee MH. Roles of p53, MYC and HIF-1 in regulating glycolysis - the seventh hallmark of cancer. Cellular and molecular life sciences : CMLS. 2008; 65:3981-3999.

91. Norris FA, Auethavekiat V and Majerus PW. The isolation and characterization of cDNA encoding human and rat brain inositol polyphosphate 4-phosphatase. The Journal of biological chemistry. 1995; 270:16128-16133.

92. Guo ST, Chi MN, Yang RH, Guo XY, Zan LK, Wang CY, Xi YF, Jin L, Croft A, Tseng HY, Yan XG, Farrelly $\mathrm{M}$, Wang FH, Lai F, Wang JF, Li YP, et al. INPP4B is an oncogenic regulator in human colon cancer. Oncogene. 2015.

93. Robey RB and Hay N. Is Akt the "Warburg kinase"?-Aktenergy metabolism interactions and oncogenesis. Seminars in cancer biology. 2009; 19:25-31.

94. Neary CL and Pastorino JG. Akt inhibition promotes hexokinase 2 redistribution and glucose uptake in cancer cells. Journal of cellular physiology. 2013; 228:1943-1948.

95. Roberts DJ and Miyamoto S. Hexokinase II integrates energy metabolism and cellular protection: Akting on mitochondria and TORCing to autophagy. Cell death and differentiation. 2015; 22:248-257.

96. Kroemer $\mathrm{G}$ and Pouyssegur J. Tumor cell metabolism: cancer's Achilles' heel. Cancer cell. 2008; 13:472-482.

97. Steelman LS, Chappell WH, Abrams SL, Kempf RC, Long J, Laidler P, Mijatovic S, Maksimovic-Ivanic D, Stivala F, Mazzarino MC, Donia M, Fagone P, Malaponte G, et al. Roles of the Raf/MEK/ERK and PI3K/PTEN/Akt/mTOR pathways in controlling growth and sensitivity to therapyimplications for cancer and aging. Aging (Albany NY). 2011; 3:192-222. doi: 10.18632/aging. 100296.

98. Fang R, Xiao T, Fang Z, Sun Y, Li F, Gao Y, Feng Y, Li L, Wang Y, Liu X, Chen H, Liu XY and Ji H. MicroRNA-143 (miR-143) regulates cancer glycolysis via targeting hexokinase 2 gene. The Journal of biological chemistry. 2012; 287:23227-23235.

99. Jiang JX, Gao S, Pan YZ, Yu C and Sun CY. Overexpression of microRNA-125b sensitizes human 
hepatocellular carcinoma cells to 5-fluorouracil through inhibition of glycolysis by targeting hexokinase II. Molecular medicine reports. 2014; 10:995-1002.

100. Peschiaroli A, Giacobbe A, Formosa A, Markert EK, Bongiorno-Borbone L, Levine AJ, Candi E, D'Alessandro A, Zolla L, Finazzi Agro A and Melino G. miR-143 regulates hexokinase 2 expression in cancer cells. Oncogene. 2013; 32:797-802.

101. Gregersen LH, Jacobsen A, Frankel LB, Wen J, Krogh A and Lund AH. MicroRNA-143 down-regulates Hexokinase 2 in colon cancer cells. BMC cancer. 2012; 12:232.

102. Wang L, Xiong H, Wu F, Zhang Y, Wang J, Zhao L, Guo X, Chang LJ, Zhang Y, You MJ, Koochekpour S, Saleem M, Huang H, et al. Hexokinase 2-mediated Warburg effect is required for PTEN- and p53-deficiency-driven prostate cancer growth. Cell reports. 2014; 8:1461-1474.

103. Yang X, Cheng Y, Li P, Tao J, Deng X, Zhang X, Gu $\mathrm{M}, \mathrm{Lu} \mathrm{Q}$ and Yin C. A lentiviral sponge for miRNA-21 diminishes aerobic glycolysis in bladder cancer T24 cells via the PTEN/PI3K/AKT/mTOR axis. Tumour biology. 2015; 36:383-391.

104. Lei M, Xie W, Sun E, Sun Y, Tian D, Liu C, Han R, Li N, Liu M, Han R and Liu L. microRNA-21 Regulates Cell Proliferation and Migration and Cross Talk with PTEN and p53 in Bladder Cancer. DNA and cell biology. 2015; 34:626-632.

105. Ou H, Li Y and Kang M. Activation of miR-21 by STAT3 induces proliferation and suppresses apoptosis in nasopharyngeal carcinoma by targeting PTEN gene. PloS one. 2014; 9:e109929.

106. Yang Y, Yang JJ, Tao H and Jin WS. MicroRNA-2 1 controls hTERT via PTEN in human colorectal cancer cell proliferation. Journal of physiology and biochemistry. 2015; 71:59-68.

107. Yang Z, Fang S, Di Y, Ying W, Tan Y and Gu W. Modulation of NF-kappaB/miR-21/PTEN pathway sensitizes non-small cell lung cancer to cisplatin. PloS one. 2015; 10:e121547.

108. Liu ZL, Wang H, Liu J and Wang ZX. MicroRNA-21 (miR21) expression promotes growth, metastasis, and chemoor radioresistance in non-small cell lung cancer cells by targeting PTEN. Molecular and cellular biochemistry. 2013; 372:35-45.

109. Wang LJ, He CC, Sui X, Cai MJ, Zhou CY, Ma JL, Wu L, Wang H, Han SX and Zhu Q. MiR-21 promotes intrahepatic cholangiocarcinoma proliferation and growth in vitro and in vivo by targeting PTPN14 and PTEN. Oncotarget. 2015; 6:5932-5946. doi: 10.18632/oncotarget.3465.

110. Li J, Jiang K and Zhao F. Icariin regulates the proliferation and apoptosis of human ovarian cancer cells through microRNA-21 by targeting PTEN, RECK and Bcl-2. Oncology reports. 2015; 33:2829-2836.

111. Xu J, Zhang W, Lv Q and Zhu D. Overexpression of miR21 promotes the proliferation and migration of cervical cancer cells via the inhibition of PTEN. Oncology reports. 2015; 33:3108-3116.

112. Xu LF, Wu ZP, Chen Y, Zhu QS, Hamidi S and Navab R. MicroRNA-21 (miR-21) regulates cellular proliferation, invasion, migration, and apoptosis by targeting PTEN, RECK and Bcl-2 in lung squamous carcinoma, Gejiu City, China. PloS one. 2014; 9:e103698.

113. Li P, Mao WM, Zheng ZG, Dong ZM and Ling ZQ. Downregulation of PTEN expression modulated by dysregulated miR-21 contributes to the progression of esophageal cancer. Digestive diseases and sciences. 2013; 58:3483-3493.

114. Eto K, Iwatsuki M, Watanabe M, Ida S, Ishimoto T, Iwagami S, Baba Y, Sakamoto Y, Miyamoto Y, Yoshida N and Baba $\mathrm{H}$. The microRNA-21/PTEN pathway regulates the sensitivity of HER2-positive gastric cancer cells to trastuzumab. Annals of surgical oncology. 2014; 21:343350 .

115. Liu J, Lei DP, Jin T, Zhao XN, Li G and Pan XL. Altered expression of miR-21 and PTEN in human laryngeal and hypopharyngeal squamous cell carcinomas. Asian Pacific journal of cancer prevention. 2011; 12:2653-2657.

116. Hopkins BD, Fine B, Steinbach N, Dendy M, Rapp Z, Shaw J, Pappas K, Yu JS, Hodakoski C, Mense S, Klein J, Pegno S, Sulis ML, et al. A secreted PTEN phosphatase that enters cells to alter signaling and survival. Science. 2013; 341:399-402.

117. Franke TF, Kaplan DR, Cantley LC and Toker A. Direct regulation of the Akt proto-oncogene product by phosphatidylinositol-3,4-bisphosphate. Science. 1997; 275:665-668.

118. Ahn KJ, Hwang HS, Park JH, Bang SH, Kang WJ, Yun $\mathrm{M}$ and Lee JD. Evaluation of the role of hexokinase type II in cellular proliferation and apoptosis using human hepatocellular carcinoma cell lines. Journal of nuclear medicine. 2009; 50:1525-1532.

119. Martelli AM, Evangelisti C, Chiarini F, Grimaldi C, Cappellini A, Ognibene A and McCubrey JA. The emerging role of the phosphatidylinositol 3-kinase/Akt/ mammalian target of rapamycin signaling network in normal myelopoiesis and leukemogenesis. Biochimica et biophysica acta. 2010; 1803:991-1002.

120. Zhuo B, Li Y, Li Z, Qin H, Sun Q, Zhang F, Shen Y, Shi Y and Wang R. PI3K/Akt signaling mediated Hexokinase-2 expression inhibits cell apoptosis and promotes tumor growth in pediatric osteosarcoma. Biochemical and biophysical research communications. 2015; 464:401-406.

121. Alvarez JV, Belka GK, Pan TC, Chen CC, Blankemeyer E, Alavi A, Karp JS and Chodosh LA. Oncogene pathway activation in mammary tumors dictates FDG-PET uptake. Cancer research. 2014; 74:7583-7598.

122. Bacci M, Giannoni E, Fearns A, Ribas R, Gao Q, Taddei ML, Pintus G, Dowsett M, Isacke CM, Martin LA, Chiarugi P and Morandi A. miR-155 Drives Metabolic Reprogramming of ER+ Breast Cancer Cells Following 
Long-Term Estrogen Deprivation and Predicts Clinical Response to Aromatase Inhibitors. Cancer research. 2016; 76:1615-1626.

123. Jiang S, Zhang LF, Zhang HW, Hu S, Lu MH, Liang S, Li B, Li Y, Li D, Wang ED and Liu MF. A novel miR155/miR-143 cascade controls glycolysis by regulating hexokinase 2 in breast cancer cells. The EMBO journal. 2012; 31:1985-1998.

124. Li Z, Li X, Wu S, Xue M and Chen W. Long noncoding RNA UCA1 promotes glycolysis by upregulating hexokinase 2 through the mTOR-STAT3/microRNA143 pathway. Cancer science. 2014; 105:951-955.

125. Geng C, Li J, Ding F, Wu G, Yang Q, Sun Y, Zhang $\mathrm{Z}$, Dong $\mathrm{T}$ and Tian $\mathrm{X}$. Curcumin suppresses 4-hydroxytamoxifen resistance in breast cancer cells by targeting SLUG/Hexokinase 2 pathway. Biochemical and biophysical research communications. 2016. 